\title{
A Streetcar Undesired: Investigating Ergonomics and Human Factors Issues in the Driver-cab Interface of Australian Trams
}

\author{
Anjum Naweed $^{1}$ (1) $\cdot$ Helen $^{\text {Moody }}{ }^{2}$
}

Received: 17 April 2015/Revised: 20 May 2015/Accepted: 9 June 2015/Published online: 13 August 2015

(C) The Author(s) 2015. This article is published with open access at Springerlink.com

\begin{abstract}
Australia is home to the biggest light rail network and the industry is currently undergoing a renaissance. However, there is littleresearch to indicate the extent to which well-informed human factors and ergonomics practises are being incorporated into tram cab design. A lack of standardised features may create transfer conflicts between cabs, as well as operational issues and concerns for occupational health. The aim of this paper is to improve our understanding of the socio-technical complexity of light rail and to enhance how design standards are informed in this domain. Various human factors methods were used, including observational cab rides, objective force assessments, interviews, and focus groups. Data were collected across two sites and analysed thematically. Analysis of data suggested a substandard level human factors and ergonomics input in the design of the cab and driver interface that violated many key tenets of established design guidelines. These were particularly concerned with the usability of the master controller (i.e. throttle lever) and various issues in the design of the tram driver workspace. Findings also revealed a number of subtle yet significant features associated with delivery of service that created safety-performance conflicts. In conclusion, very little human factors input of tram driving, and the ergonomics considerations of the driver's workplace in general, appear
\end{abstract}

Anjum Naweed

anjum.naweed@cqu.edu.au

1 Appleton Institute for Behavioural Science, Central Queensland University, 44 Greenhill Rd, Wayville, SA 5034, Australia

2 Injury Prevention and Management, Corporate Health Group, Adelaide, SA, Australia

Editor: Marin Marinov to be going into the design of tram cabs. This may be related to the practice of using non-specific standards for developing trams and/or poorly integrating human factors and ergonomics into their specification processes. Some considerations for future work are given.

Keywords Modern tram - Traffic and transport safety . Light rail · Interdisciplinary transportation research · Cab design $\cdot$ Interface design

\section{Background}

Australia has over $270 \mathrm{~km}$ of tramway and is home to the biggest and oldest urban light rail network in the world [1]. There are also signs that the Australian light rail industry is undergoing a renaissance; new light rail projects are being planned, are under construction, or currently operational in Melbourne, Adelaide, Canberra, Perth, Newcastle, Sydney, and the Gold Coast [2, 3].

Much of the published literature on Australian Trams has tended to explore its history, growth, and design, specifically in terms of its engineering [e.g. 1, 4]. Marketing brochures for modern trams typically advertise innovations in technology, including advanced bogie designs, provision of passenger amenities, and modularised car-body components for collision safety and ease of repair [5]. Some emerging classes have also won design awards [6]; however, very little published research indicates the extent to which good ergonomics and human factors (E/ $\mathrm{HF}$ ) principles have been incorporated into the design of tram cabs. This space typically falls into the design and consultation space of rail manufacturing companies that service the industry. In recent years, the absence of this has been made apparent by its coverage in the media spotlight 
for reports of work-related injury and stress reducing the drivers' ability to work [7].

Beyond occupational health and safety concerns, there are a number of reasons why the tram system would be important from an E/HF perspective. First, many tram classes operate in Australia's individual states and increasing the number of classes of trams on a single network invariably decreases the range of standardised features. This has the effect of creating inconsistencies in stimulus-response design and may lead to conflicts when transferring between different trams [8], particularly if there has been no consideration to matching and/or retrofitting frequently used features. Additionally, the lack of standardisation may create variations in the design of the cab interface and impact on driving performance. For example, injuries associated with passengers falling in the saloon are among the most common safety issue [9] and linked with concerns for jerky driving [10]. Whilst some of these issues may be related to human performance and training, it is worth questioning the extent to which they are influenced by problems in tram cab design.

Most of the functional requirements for designing the driver-cab of a tram appear to have been extracted from standards originally written for generic locomotives and driving coaches [e.g. 11]. Although the tram and train driving tasks have synergies, heavy, and light rail are fundamentally different systems, and the resulting designs may not always be suitable. The operation of trams in mixed-road traffic environments (i.e. as streetcars or street moving vehicles) stretches this gap further. Theories that orientate towards systems design such as the joint-cognitive systems movement suggests that drivers are likely to have a very unique dynamic with their vehicles, and an inter-connectedness that means they operate with it as an intimate system [12]. Based on this notion, one may assume that any differences in the features and characteristics of the two environments are likely to create subtle but significant differences in task dynamics. This is one of the reasons why it is important to involve the end-user in participative design processes, so as to ensure that the system, interface and the task work and behave as intended [13].

Norman's six design principles, which still underpin thinking around good design theory, are based on visibility, feedback, affordance, mapping, constraint, and consistency $[14,15]$. In terms of tram cab design, these can be considered to correspond with: whether drivers can see the state of the tram, and if controls are positioned in a way that can be easily found and used (visibility); whether drivers know tram state, what it is doing, and what action needs to be performed (feedback); whether the perceived and actual properties of the various control and functions of the tram clearly elucidate their operation (affordance); whether there is a clear relationship with controls and their effect (mapping); whether there are any clear restrictions to the kind of interactions that can take place (constraints); whether cab-interfaces are designed to have similar operations; and whether systems are learnable and drivers can quickly transfer prior knowledge to new contexts (consistency). One would not expect many of these tenets to be violated or substantially compromised in the design of modern trams.

Industrial ergonomics literature has identified $\mathrm{E} / \mathrm{HF}$ issues in the design of tram cabs and suggested improvements, particularly for the primary speed/brake throttle controller (also known as the master controller), and "deadman" safety features (i.e. safety devices that operate if the driver is incapacitated) [16]. The general expectation is that once cab design issues have been identified, improvements will filter back into the specifications during the tendering and bidding process for new rolling stock classes. However, there are few publically accessible ways of tracking how, when, and if this happens. In general, new knowledge or insights are also expected to inform standards, but this process does not always translate either.

\subsection{Aims}

The aim of the research presented into this paper is to improve our understanding of the socio-technical complexity of light rail, and how design standards are informed in this domain. To fulfil the aims of this research, the objective of this paper is to draw on established design theory [14] to provide an account of the key E/HF issues in cab and task design that have been observed in the Australian tram industry. Whilst we share insights that would be of interest to the academic community, our goal is to provide a platform for debate and discussion for academic and industry practitioners, particularly those within the engineering urban rail transit community.

\section{Method}

\subsection{Research Context}

The research reported in this article was undertaken at two different sites (i.e. two different light rail networks) in Australia. The work was initiated by tram operators in response to complaints and concerns provided by tram drivers. In one site, this followed a very high incidence of musculo-skeletal injuries with particular incidence of left shoulder/arm injuries, and in other, through reports of cab design issues in the driver-cabs of newly deployed rolling stock. 


\subsection{Design}

Several methods were converged to evaluate and assess the workplace of the tram driver. These fell into a mainstream range of qualitative techniques that are commonly used to evaluate humans at work, and included cab walkthroughs on stationary trams, on-road observations on scheduled services, objective force assessments, interviews, and focus groups.

Converging multiple data collecting techniques has been recommended as an effective means of eliciting knowledge [17] and performed successfully on other rail research [e.g. 18, 19]. Undertaking the work in different sites provided the means to compare common issues across different tram classes and identify the scope of specific E/HF problems. The methodology also included a specific physical ergonomics assessment and a broader human factors investigation. Figure 1 shows an overview of the methodology for each of these components, including individual methods of data collection and the main areas of focus. As Fig. 1 shows, unstructured interviews, cab walkthroughs, and cab rides were common to both components.

The physical ergonomics work assessed the driver's interaction with their cab in terms of how the interface fitted their body and addressed their functional needs. Methods in this component included unstructured interviews, cab walkthroughs (on stationary trams), and cab rides. Unstructured interviews were conducted with groups of drivers in the tram depot, or entirely opportunistically with individual drivers during cab walkthroughs and cab rides. Cab walkthroughs were pre-planned and involved a detailed discussion of the cab with a driver trainer who "walked" through the layout of the various controls, described their functions, and demonstrated how they were operated. The process lasted 30-60 min. Responses to questions were voice recorded, and photos were taken. During the walkthroughs, cabs were also assessed in terms of the push-pull forces and static muscle load required to operate controls. Particular focus was placed on the master controller and "deadman" devices, and measurements were taken using a Mecmesin force gauge. Cab rides were pre-planned "out and back" journeys from the main depot, undertaken during scheduled services (i.e. with passengers) but also out of service. These lasted 30-120 min and included spells of interviewing and silent observation of the driver engaging with their task.

The human factors work examined the driver-cab in terms of the design of the tram-driving task itself and its cognitive demands. The methods in this component also included cab walkthroughs, driving observations, and unstructured interviews, and these were undertaken as previously described. However, this component also included a series of focus groups. These lasted $1.5-2 \mathrm{~h}$, conducted with 4-6 tram drivers per group, and included a scenario simulation task [20]. This task required each of the participants to create challenging driving scenarios, as a means to overcome conversation-based limitation, but also stimulate situational insight. These methods has been previously applied in the heavy rail industry and have been very effective for identifying specific cognitive task demands, and illustrating how driver's stabilise the task in the face of conflicting goals [20]. Example questions for the general focus group were "What are your thoughts on the [specific class] tram?" and "take me through a challenging part of a route that you drive over." Example questions for the scenario simulation task were "create a really hard stretch of track" and "imagine you have to drive over your route. List the strategies that you would use to navigate it."

\subsection{Participants and Recruitment}

Participants provided informed consent to take part in the work, but in all cases, contacts at the relevant organisations were required to mediate and facilitate access. Whilst most activities were pre-planned, given the nature of the fieldwork, this was subject to change.

\subsection{Data Collection Decomposition}

Our work comprised assessment of two specific tram-types from two different rail manufacturing companies servicing the industry. Our assessments were related to prior incidence of musculo-skeletal injury in one tram, and a concern for the skills transfer issues in the other. In both cases, issues were first raised and reported by tram drivers. A further four tram classes were observed in order to create a basis for cross-comparison, making six tram classes in total. As two of these classes comprised observations of an earlier and later model (of the same class), eight different trams were actually observed across both of the sites.

Figure 2 decomposes the data collection in each of the sites by showing the number and duration of each of the methods and the number of drivers that took part. Six cab rides with a total of $7 \mathrm{~h}$ of observation were performed. The unstructured interviews were undertaken with 10 drivers. As shown in Fig. 2, the physical ergonomics assessment was only performed at one site, though this was on two tram classes common to both. The focus groups were also undertaken in one site, though this was the larger network of the two with a much greater variety of tram types.

\subsection{Data Analysis}

Like the E/HF methods used to collect data, the analysis was largely qualitative. For the ergonomics assessment, 
Fig. 1 Overview of the methodology

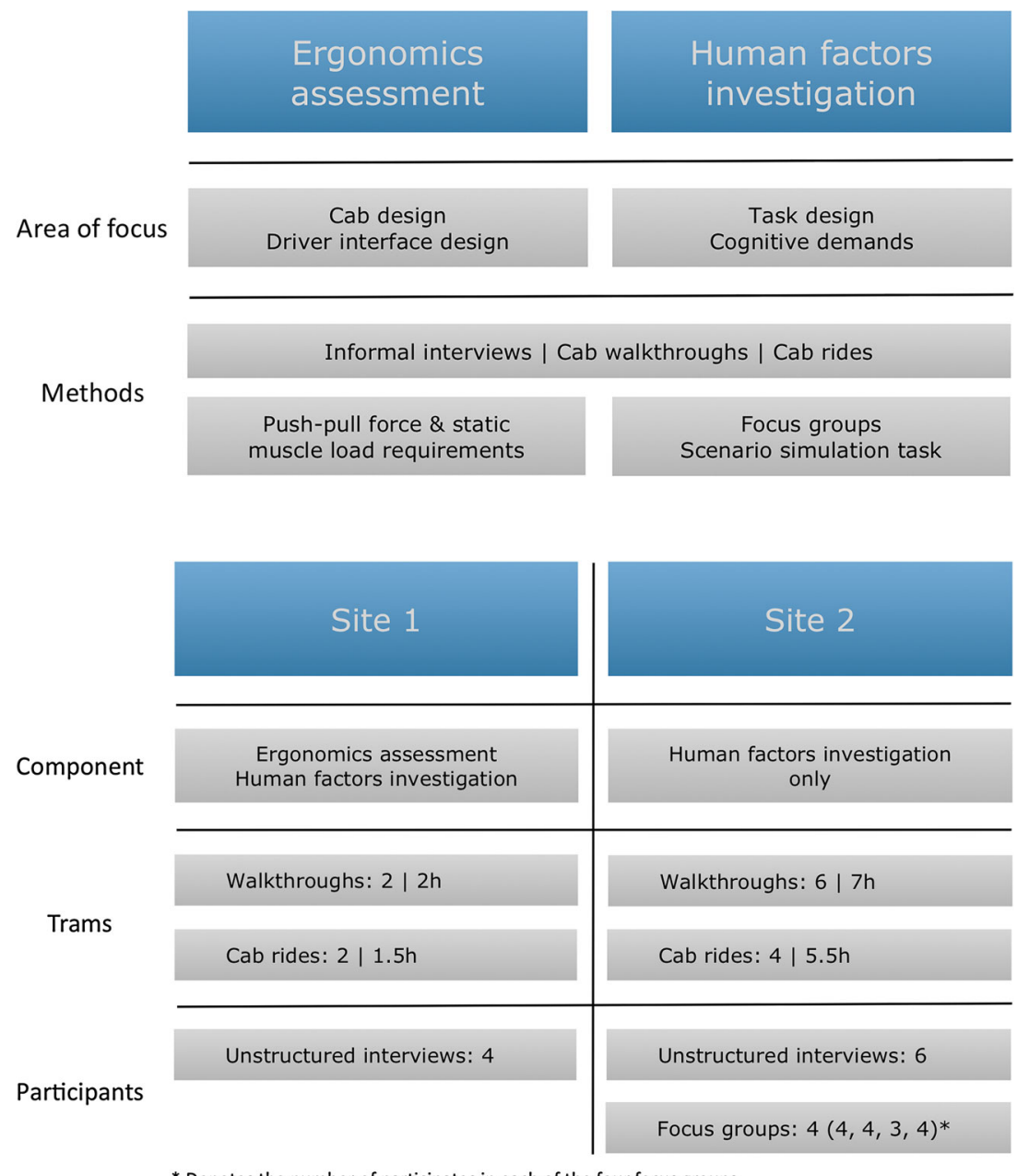

* Denotes the number of participates in each of the four focus groups
Fig. 2 Decomposition of activities in each of the data collection sites reach agreement on the codes that were used. This analysis was undertaken in multiple rounds and included a process of constant comparative analysis and cross-data validity check to determine consistency of the analysis and allow for further refining $[22,23,24]$. To achieve this, the data were checked regularly against other sources, such as photos of different cab designs from the various classes, scenarios from the focus groups, and injury statistics. The groupings and emerging themes were then checked with subject matter experts and tram drivers (i.e. end-users) in the participating organisations $[25,26]$.

\section{Results}

Before presenting findings from the thematic analyses, we will set the context by briefly describing our observations of the driver-tram system in terms of the type of cognitive work involved in the task. After this, we will provide three themes that appeared to evidence very little specification of $\mathrm{E} / \mathrm{HF}$ considerations in the design of the cab for human use. 
The themes comprised design considerations associated with the cab, the driver-cab interface, and the task.

\subsection{The Driver-Tram System}

The driver and the tram operated in a transportation system that was expressed in terms of how well-traffic moved. There were signals, signs, points (i.e. switches that selected the route), and rules of the road, all of which defined issues of flow and required technical knowledge. Most of the observed trams served as a streetcar in the main; that is, they moved on the road with adjoining vehicles but also on their own rail corridors (i.e. a dedicated light rail corridor section). Some sections of track necessarily had less tram and road vehicle separation, largely as a result of increased passenger densities. The task of tram driving itself was observed to have a number of distinct features. First, it was highly dynamic, both in terms of the number and frequency of tasks needing to be performed. The consensus from participants was that they performed three core tasks: (1) preparing the tram so it was safe before starting the shift; (2) ensuring a smooth journey; and (3) providing good customer service within the time constraints. These goals were closely coupled and expertise was characterised by the ability to regulate them well. Trams could move too fast or too slow with little effort, meaning that they could encroach easily beyond points of no return with relative ease. This included road cross hatchings representing "no mans land" with little clearance for turning trams, going through red lights into road traffic, or driving over points that were set incorrectly.

The complexity in the task was defined by the goals of comfort (braking and accelerating smoothly), time-accuracy (maintaining the stopping pattern), and regulating speeds effectively. Thus, the task incorporated an autonomously driven process on two dimensions: first, the driver controlled the acceleration and braking of the tram, not the speed itself; and second, there was a time requirement to meet with service delivery imposed at higher levels. Analysis of the task revealed six main subtasks, each of which was directly related with one or more of the three overarching goals. These are shown in Fig. 3. All but one (vigilance of the road and its users) was directly related to the three core tasks.

Throttle and braking was complex and drivers needed to accurately estimate the influence of gradients and changing conditions on speed in order to do it efficiently. Tram handling was subject to variation as a result of changes to wheel adhesion brought on by changes in weather. Whilst drivers could deploy sand to improve adhesion, some slipinducing agents were not always visible (e.g. millipedes). Thus, much like train driving, tram drivers spoke of driving by the "feel of the tram" and by "instinct" highlighting the role of non-technical skills. The driver in the driver-tram system needed to be attentive, vigilant, and remain highly aware of the environment as it evolved around them. They also needed to think and react very quickly, but as a human, they were susceptible to fatigue and psychological impacts associated with shift work. It was also easy to get distracted by sources related to the task, such as time pressure, as well as non-task-related sources, such as passenger chatter. The final element of the system were the goals that informed decision-making processes. The driver needed to regulate safety against productivity, but in practice, pressures and other motivations influenced this process. Cultural norms within the organisation and environment (e.g. road-user behaviour), and other social influences meant that the way a driver operated a tram in one site was likely to be very different from another.

\subsection{Cab Design: Confusion Hath Now Made his Masterpiece $^{1}$}

Multiple classes and types of tram operated on the networks represented by both sites. Numerous differences in cab design were observed for trams operating on the same network, attracting potential for conflicts when moving between cabs. In one tram for example, sounds were easily masked by other alarms. In another tram (on the same network), the alarms were very loud, such that they frequently "startled" the driver. For example, participants considered the buzzer associated with vigilance checks on one tram to be overly loud, stress inducing, and resonate in the ears long after it had gone. These issues illustrated problems in the design of auditory icons, inappropriate urgency and hazard matching of cab alarms, and ultimately, design issues with external consistency. Although the perception of sound was based on subjective assessment, they appeared to contravene recommended noise design levels (i.e. no greater than $85 \mathrm{~dB}(\mathrm{~A})$ for any length of time and $15-25 \mathrm{~dB}(\mathrm{~A})$ above this) for alarms [27]. Problem or difficulties with inconsistency are usually a result of different systems rarely observing the same design standards [14].

Issues were also observed with the design and placement of buttons, such that participants confused some of them during walkthroughs. Figure 4 shows a tram cab where eight buttons were integrated into the armrest of the driver seat. The buttons showed very low discrimination in design - that is, they were identical to feel and touch, which increased head-down activity during driving. They also had no backlighting, which created problems in low light/night driving. To overcome this, some drivers indicated that they "pulsed the [cab] light" to improve

\footnotetext{
${ }^{1}$ Shakespeare, W. (In 2.3. Macbeth).
} 
Fig. 3 Overview of the six subtasks associated with three core tasks in tram driving

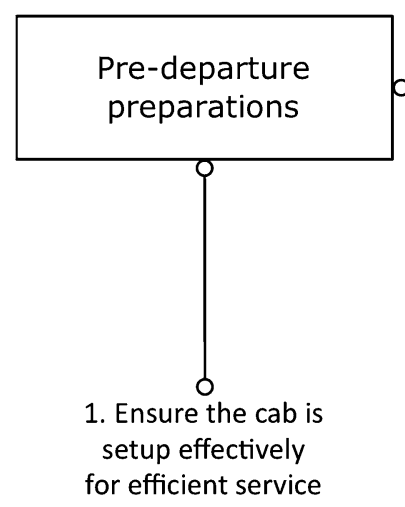

setup effectively

for efficient service

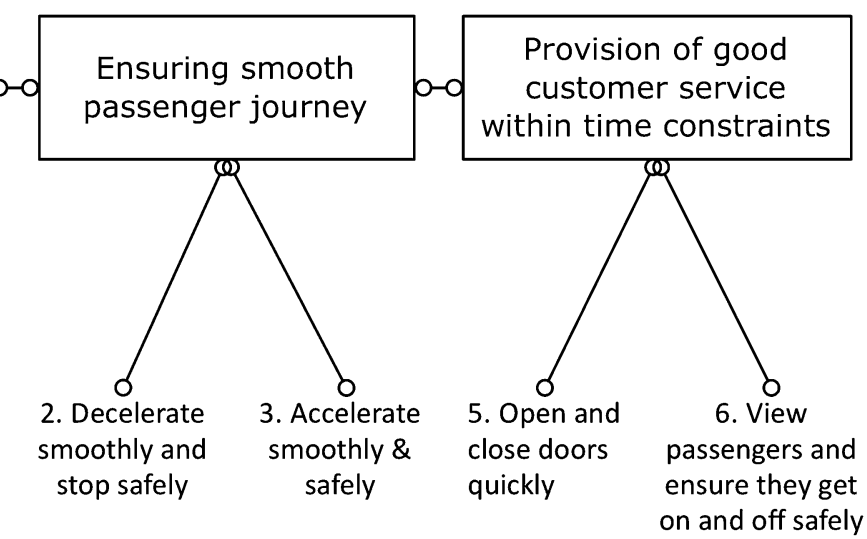

4. Be vigilant of the road and its users visibility whilst others used a torch. Both of these strategies increased task loading, but the problem was exacerbated by light reflecting off the windscreen, which restricted visibility even further. Generally speaking, there was no common design language in the quantity and clustering of buttons. In focus groups, few participants who operated this tram were able to recall the exact location and position of buttons, though some indicated that cab designers had consulted a small reference group of tram drivers as part of the design process. The categories of findings in the cab design theme reflected several departures from Norman's [14] design principles, particularly with visibility, feedback, mapping, constraints, and consistency.

\subsection{Interface Design: Action is Eloquence ${ }^{2}$}

The master controller was the primary means of controlling the tram and integrated throttle and braking commands into a single lever. These were mapped to the direction of travel (i.e. moving the master controller forward moved the tram forward). Participants described a process of working with the master controller to understand how throttle manipulations influenced tram speed. This was a non-technical skill and descriptions supported the joint-cognitive systems view [12]. The master controller is ultimately associated with the safety function of ensuring a smooth passenger journey task and subtask, as shown in Fig. 3. The problems observed with the master controller fell into a number of categories and included designs with low correspondence between body and arm posture, variation in reach distances, and forearm pads that did not facilitate contact with all master controller notch positions. For example, the master controller in Fig. 4 was integrated into the right-arm of the driver seat and could only be adjusted by pivoting the armrest up or down at the point it joined the rear of the chair. The way that master controller movements graduated

\footnotetext{
${ }^{2}$ Shakespeare, W. (In 3.2. Coriolanus).
}

with accelerating and braking also varied between trams, within and between the two sites. Predicting changes in tram speed was therefore less reliable and reported as a contributing factor to work-related stress.

Some master controller designs required application of sustained push force via spring-loading; that is, they were designed to fall back into the brake position if the driver were to let go (i.e. a "deadman" device). One master controller required a static force exertion that exceeded the recommended duration, and as such, was considered to be the main cause for incidence of musculo-skeletal injuries in that tram. During cab rides, we observed frequent changes in the hand positions of participants who operated this type of master controller (see Fig. 5). The same drivers did not show these in conscious demonstrations, suggesting that the process occurred enactively [28] (i.e. operated outside of conscious awareness), likely as a strategy to distribute physical loading and fatigue across different muscle groups.

Most rail-based systems use features that perform regular "vigilance" checks. According to standards for generic locomotives and driving coaches, these devices must be capable of stopping the tram in the event that the driver is incapacitated [11]. For this reason, they are usually designed with a contact-point (e.g. a button) that requires frequent contact; the idea being that it acts as a safety measure in the event of physiological breakdown (e.g. from malaise, sleep). In one tram, the maximum holding time for one of these devices was $12 \mathrm{~s}$ with $3 \mathrm{~s}$ for maximum release. Thus, if the driver held down the button for more than $12 \mathrm{~s}$ (without carrying out an action) or released it for longer than $2 \mathrm{~s}$, a buzzer sounded after which the driver had $2 \mathrm{~s}$ to carry out an action to prevent the emergency brakes from activating.

The timing given to respond to these devices varied for different trams, between and within the two sites, meaning that the driver had much longer to respond to the device in one type of cab than in another. The mechanism used to 
Fig. 4 Buttons that have been integrated into the armrest of the cab. Left photo shows whole seat with buttons (circled); right photo shows a close-up
Fig. 5 Matrix of photos showing examples of variation in hand positions observed when operating a master controller that required sustained push force
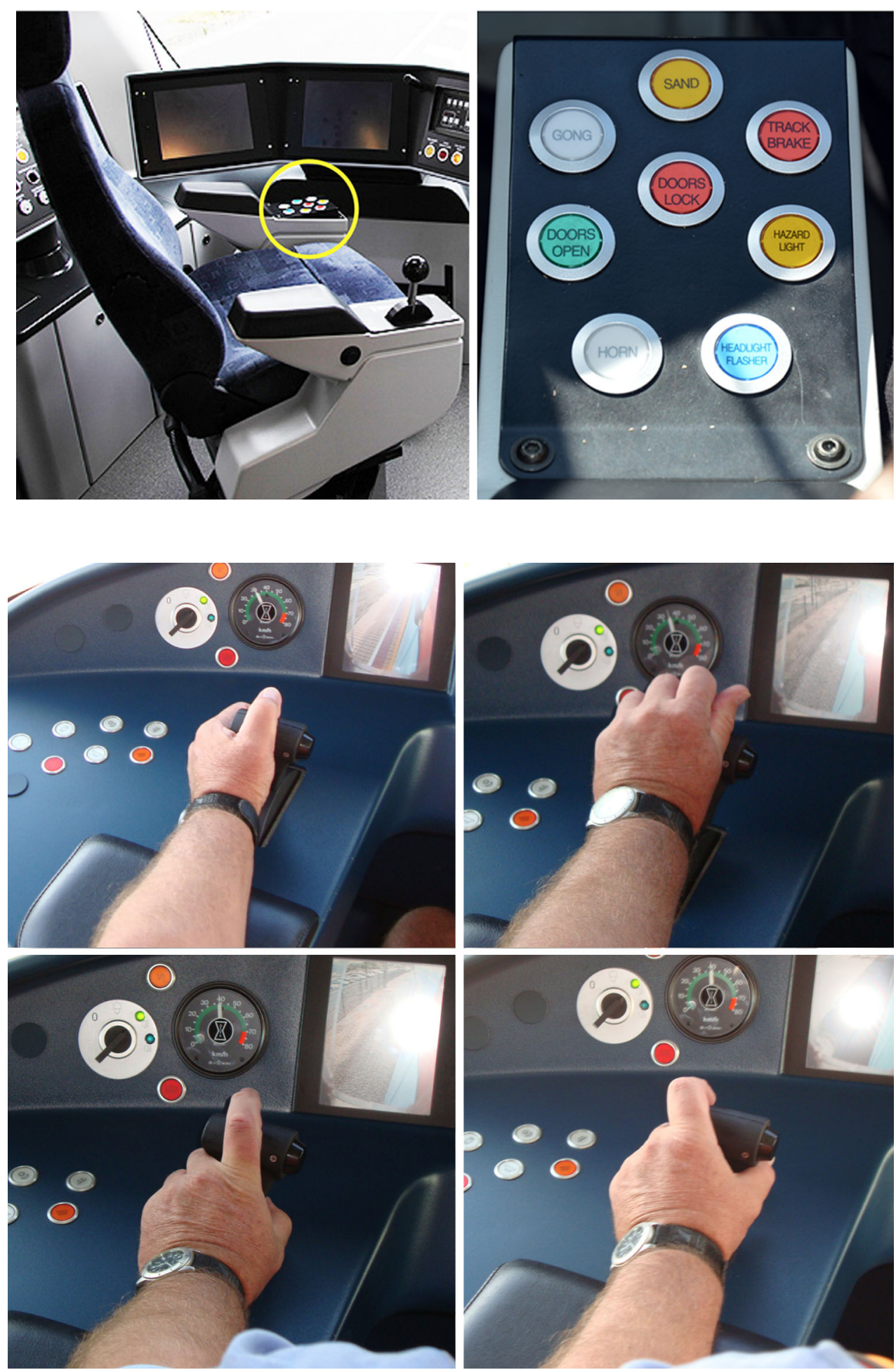

reset the device also varied. In some it was a foot pedal, in others, a sensitive thumb button/contact, and others had both. In some trams, the driver could not swap between the thumb and foot even though cab design indicated this possibility. In one tram, the system required drivers to touch a sensor on the master controller with their thumb, but it was reported to have poor responsiveness. During cab rides, it was observed being tapped and swiped, or stroked regularly in clockwise and counter-clockwise motions. This behaviour increased task loading, but also appeared to be a habituated process used to overcompensate for the warning noise (see Cab design theme) and prevent it from 
sounding. Analysis from the interface design theme identified a number of departures from Norman's design principles [14], namely, feedback, mapping, and consistency.

\subsection{Task Design: Time is Out of Joint ${ }^{3}$}

Tram driving requires the drivers to wrestle between safety and productivity goals. For some drivers, this generated time pressure. Although this was considered a norm for the task, the experience was intensified by the design of the vigilance task. The focus on time had the effect of taking attention away from other tasks, such as stopping the tram smoothly, opening and closing doors efficiently, and confirming that passengers were getting on and off safely. Further, the design of the task reflected these tensions; as an example, the doors on the trams had sensors, which prevented them from being closed if they were obstructed (e.g. by a person, bag, push-chair). However, the trams also had a force-door close button, which could override the passenger detection sensors and close the door. Many drivers confessed to using this button over the regular door close button, in spite of organisational policy.

In newer trams, drivers were presented with a digital time-keeping performance indicator in their interface to show how fast or slow they were moving between stops. This information was updated in real time as a pseudoestimated time of arrival or ETA feature. Indeed, some participants were observed using these changes in time to regulate the master controller (and therefore tram speed) instead of the information displayed in the speedometer. Thus for some drivers, the time shown in this device was being used to parameterise their speed choices instead of the speeds on the road. Performance penalties for the tram industry were reportedly associated with being too early than for being too late. This was not surprising, given the focus on maintaining flow and minimising impact to other services. At one site, penalties were administered if the driver was more than 1-min early or more than 6-min's late. This was considered to create a "hurry up and wait culture," which emphasised time keeping and anxiety from lack of control in work pacing. This effect is supported by observations in the train system when station dwelling [20], but is of particular concern in the tram context, given that time pressure has been associated with negative emotions, stress, and the increased propensity for risk taking behaviours on roads $[23,29]$. The theme of task design revealed a number of breaks from Norman's design principles, namely affordance (i.e. using changes in time from the ETA feature to change speed), mapping, and constraints.

$\overline{3}$ Shakespeare, W. (In 1.5. Hamlet).

\section{Discussion}

The E/HF issues we observed suggested to us that the standards being used to inform the design of older trams, as well as newer ones, may not have been specified well enough for the socio-technical complexity in the system. This was clearly evidenced in the three themes where there were multiple departures and compromises in good-practice design [14]. Most of the trams we observed were modern trams, one of which was undergoing commissioning and acceptance at the time of the research. Thus, they suggested that whilst standards were adequately specified, the process of consultation and end-user design was lacking. They may also have pointed to problems in translating standards into practice. The lack of involvement from $\mathrm{E} / \mathrm{HF}$ designers and/or good $\mathrm{E} / \mathrm{HF}$ practises in the design of the trams, particularly in their early stages, is likely to be a contributing factor. However, as designers, managers, and suppliers involved in the tram procurement process were not interviewed as part of the work, this is difficult to substantiate. If these issues are indeed part of the problem, it is important to note that inviting the authors to conduct these assessments is a sign of change. Participants indicated that tram drivers (i.e. end-users) had actually been consulted during the early part of the design process - particularly with respect to data presented in the theme of cab design, and there was consensus to support this. However, given the observed issues with the resulting design (e.g. placement of buttons in the driver seat), the process is likely to have occurred at the cost of expert E/HF input - that is, the tram drivers could have been consulted under the premise that they were experts in design as well as experts in tram driving.

Many of our observations and findings pointed to important and relevant concerns of the impacts of cab design on work and stress, but there were also data to support this from the perspective of task design. There were strong indications that participants experienced time pressure and anxiety associated with regulating safety goals, such as driving smoothly, letting passengers on and off efficiently, and with their productivity goals, such as keeping to the schedule. These tensions may filter through to the task in the form of suboptimal (i.e. jerky) journeys, and door opening issues; therefore it is useful to monitor these events as a measure for risk. Key observations for $\mathrm{E} / \mathrm{HF}$ communities, and standards design authorities were as follows:

- Inefficiencies or issues in vigilance device for the cab, interface, and task design spectrum

- Distraction and inattention to the tram-driving task from time pressure and over-emphasis on productivity goals 
- Inordinately high forces required to operate the master controller

- Lack of forearm support when operating the master controller

- Inconsistencies in noise, particularly for auditory alarms

- Difficulties for operators to achieve the correct posture due to a combination of poor seating adjustments and console/cabin design.

\subsection{Methodological Review}

Human Factors integration is concerned with "providing a balanced development of both the technical and human aspects of equipment procurement" and essentially "ensures the application of scientific knowledge about human characteristics through the specification, design and evaluation of systems." [30, p. 6] The key advantage of the methodology used in this paper is that it was driven by a multidisciplinary approach, combining the expertise of mechanical engineers, human factors specialists, and ergonomists.

The methods that were used in the study substantially increased the ability for the investigators to understand the problems. A combination of discussions, generative simulation tasks, and observations of enactive behaviours onand off-the-job enabled the participants to more easily describe their own knowledge and thought processes, but also overcame the problem of any inaccuracies of selfreporting from memory and decision processes [31]. As a qualitative process, the study provided a richness and depth, particularly given the integration of objective force assessments. However, the methods could be further strengthened by the addition of suitable quantitative methods (e.g. national surveys, simulator work).

\subsection{Further Research}

For the rail industry, considerations into the redesign of seating, the master controller, and the foot pedal are advocated to achieve more flexibility and correct posture for tram drivers when driving. Work is currently being undertaken in one site involving cab design modifications to determine optimum driver interface with the master controller and foot pedal based on the findings to date with the aid of mock-ups. This will be the subject of further reporting when the testing is completed. Further academic research should also consider the specific makeup of the tram-driving task using task analyses. Towards achieving this aim, more analysis of the data collected in this work may be used to develop a framework for further analysis, specifically in terms of how the task demands interact with the specific ergonomic and human factors issues.

The findings evidence a general need to investigate any strategies for reducing risk-related stress in this population. This may start by examining the informal strategies to manage and mitigate stress associated with safety risk and performance conflicts. Lastly, it would be useful undertake more work to determine if and how suggested improvement in cab ergonomics filter back into tram classes when specifying bids, tenders, and contracts for new rolling stock.

\section{Conclusions}

A combination of physical ergonomics assessments and human factors investigations identified issues with design and tram operation in two sites in Australia. The findings suggest a dearth of ergonomics and human factors considerations of the design of the driver interface and the cab. This may be related to the practice of using non-specific standards for developing trams and/or poorly integrating human factors into their specification processes.

Acknowledgments The authors gratefully acknowledge the anonymous operators and contractors that invited the authors to assess their tram cabs. They are also very grateful to Ganesh Balakrishnan for his assistance.

Conflict of interest The authors believe there are no conflicts of interest (financial or otherwise) that impinge on the quality or impartiality of the research in the paper.

Open Access This article is distributed under the terms of the Creative Commons Attribution 4.0 International License (http://crea tivecommons.org/licenses/by/4.0/), which permits unrestricted use, distribution, and reproduction in any medium, provided you give appropriate credit to the original author(s) and the source, provide a link to the Creative Commons license, and indicate if changes were made.

\section{References}

1. Currie G, Burke M (2013) Light rail in Australia-performance and prospects. Paper presented at the Australasian Transport Research Forum, Brisbane, Australia

2. Australasian Railway Association (2015) Capital metro: Canberra's light rail project in a global context. Author, Canberra

3. Nye B (2015) Light rail in Australasia: the economic, social and environmental case. In: Proceedings of 2015 light rail conference, NSW, AU March 5-6 2015

4. Macdonald A, Coxon S (2011) Towards a more accessible tram system in Melbourne-challenges for infrastructure design. Paper presented at the Australasian Transport Research Forum, Adelaide, Australia

5. Alstom Transport (2011) What will your Citadis be? Author. http://www.alstom.com/transport/products-and-services/trains/tram way-citadis/ 
6. Good Design Australia (2014) Good design awards 2014. Crowther Blayne, Surfers Paradise

7. Carey A (2012,) Tram cop a low blow as report slams design flaws. The Age (July 14)

8. Wickens CD, Hollands JG (2000) Engineering psychology and human performance, 3rd edn. Prentice-Hall, Upper Saddle River

9. Mitra B, Al Jubair J, Cameron PA, Gabbe BJ (2010) Tram-related trauma in Melbourne, Victoria. Emerg Med Australas 22(4):337-342. doi:10.1111/j.1742-6723.2010.01309.x

10. Middendorp C (2010) Hop on tram for on hell of a scary ride. The Sydney Morning Herald, December 23

11. Union Internationale des Chemins der fer (2009) Driver machine interfaces for EMU/DMU, locomotives and driving coachesfunctional and system requirements associated with harmonised Driver Machine Interfaces. Author, Paris, France

12. Woods D, Hollnagel E (2006) Joint cognitive systems: patterns in cognitive systems engineering. Taylor \& Francis, Boca Raton

13. Gould JD, Lewis C (1985) Designing for usability: key principles and what designers think. Commun ACM 28(3):300-311

14. Norman D (1988) The design of everyday things. Basic Books, New York

15. Preece J, Rogers Y, Sharp H (2002) Interaction design: beyond human-computer interaction. Wiley, New York

16. Foot R, Doniol-Shaw G (2008) Questions raised on the design of the "dead-man" device installed on trams. Cogn Technol Work 10(1):41-51

17. Cooke NJ (1994) Varieties of knowledge elicitation techniques. Int J Hum Comput Stud 41(6):801-849

18. Naweed A (2014) Investigations into the skills of modern and traditional train driving. Appl Ergon 45(3):462-470. doi:10.1016/ j.apergo.2013.06.006

19. Naweed A, Balakrishnan G, Bearman C, Dorrian J, Dawson D (2012) Scaling generative scaffolds towards train driving expertise. In: Contemporary ergonomics and human factors 2012, pp 235-236
20. Naweed A (2013) Psychological factors for driver distraction and inattention in the Australian and New Zealand rail industry. Acc Anal Prev 60:193-204. doi:10.1016/j.aap.2013.08.022

21. Huberman MA, Miles MB (1994) Data management and analysis methods. In: Denzin NK, Lincoln YS (eds) Handbook of qualitative research. Sage, Thousand Oaks, pp 209-219

22. Charmaz K (2006) Constructing grounded theory: a practical guide through qualitative analysis. SAGE Publications Ltd, London

23. Naweed A, Rainbird S, Dance C (2015) Are you fit to continue? Approaching rail systems thinking at the cusp of safety and the apex of performance. Saf Sci. doi:10.1016/j.ssci.2015.1002.1016

24. Naweed A, Balakrishnan G, Bearman C, Dorrian J, Dawson D (2012) Scaling generative scaffolds towards train driving expertise. In: Anderson M (ed) Contemporary ergonomics and human factors 2012: proceedings of the international conference on ergonomics \& human factors 2012. CRC Press, Blackpool, p 235-236

25. Powell C (2003) The Delphi technique: myths and realities. J Adv Nurs 41(4):376-382

26. Hsu CC (2007) The Delphi technique: making sense of consensus. Pract Assess Res Eval 12(10):1

27. Edworthy J, Hellier E, Noyes J, Aldrich K, Naweed A, Metcalfe GR (2008) Good practice guide for the design of alarms and alerts. Rail Safety Standards Board, London

28. Branton P (1979) Investigations into the skills of train-driving. Ergonomics 22(2):155-164

29. Cœugnet S, Naveteur J, Antoine P, Anceaux F (2013) Time pressure and driving: work, emotions and risks. Transp Res Part F 20:39-51. doi:10.1016/j.trf.2013.05.002

30. MoD (2000) Human factors integration: an introductory guide. HMSO, London

31. Bisantz A, Roth (2008) Analysis of cognitive work. In: BoehmDavis DA (ed) Reviews of human factors and ergonomics. Human Factors and Ergonomics Society, Santa Monica, pp 1-43 